\title{
Electronic Properties of Mononuclear, Dinuclear, and Polynuclear Cobaltacarboranes: Electrochemical and Spectroelectrochemical Studies
}

\author{
Fabrizia Fabrizi de Biani, Maddalena Corsini, Piero Zanello* \\ Dipartimento di Chimica, Università di Siena, Via A. Moro, 53100 Siena, Italy \\ Haijun Yao, Martin E. Bluhm, Russell N. Grimes* \\ Department of Chemistry, University of Virginia, Charlottesville, Virginia, 22901
}

\section{Supporting Information}

Synthesis and characterization of 4, 7a, and 14-17.

Instrumentation. ${ }^{1} \mathrm{H}\left(500 \mathrm{MHz}\right.$ (where noted), 300MHz), ${ }^{13} \mathrm{C}(125.8 \mathrm{MHz}$ (where noted), 75.4 MHz), and ${ }^{11} \mathrm{~B}(96.4 \mathrm{MHz}) \mathrm{NMR}$ spectra were recorded on GN-300/44 instruments. ${ }^{1} \mathrm{H}$ and ${ }^{13} \mathrm{C}$ shifts are referenced to residual ${ }^{1} \mathrm{H}$ and ${ }^{13} \mathrm{C}$ signals in the deuterated solvent. ${ }^{11} \mathrm{~B}$ NMR resonances are referenced to the external standard $\mathrm{BF}_{3} \bullet \mathrm{OEt}_{2}$. Unit resolution mass spectra were acquired on a Finnigan (Model LCQ Classic) quadruple ion trap mass spectrometer using an atmospheric pressure chemical ionization interface. Infrared spectra were obtained on a Nicolet Impact-400 spectrophotometer. Ultraviolet-visible spectra were recorded on a HP 8452A diode array spectrophotometer or Cary 5E UV-VIS-NIR Spectrophotometer. Elemental analyses were performed by Atlantic Microlab in Norcross, GA.

Materials and Procedures. All reactions were carried out in oven glassware under a dinitrogen atmosphere using conventional glove box or Schlenk techniques, and the products were worked up in air. All commercial reagents were used as received without further purification. THF was distilled from sodium benzophenone ketyl prior to use. Triethylamine was distilled from $\mathrm{CaH}_{2}$ under an inert atmosphere. When anhydrous dichloromethane was required, the solvent was distilled from $\mathrm{P}_{2} \mathrm{O}_{5}$ under $\mathrm{N}_{2}$. Cp* $\mathrm{Co}\left(2,3-\mathrm{Et}_{2} \mathrm{C}_{2} \mathrm{~B}_{4} \mathrm{H}_{4}\right),{ }^{1} \mathrm{Cp} * \mathrm{Co}\left(2,3-\mathrm{Et}_{2} \mathrm{C}_{2} \mathrm{~B}_{3} \mathrm{H}_{5}\right),{ }^{1}$ $\mathrm{Cp} * \mathrm{Co}\left(2,3-\mathrm{Et}_{2} \mathrm{C}_{2} \mathrm{~B}_{3} \mathrm{H}_{3}-4,6-\mathrm{I}_{2}\right),{ }^{2}$ (A1) and $(\mathrm{Cp} * \mathrm{CoCl})_{2}{ }^{3}$ were prepared according to published procedures.

$\left[\mathbf{C p} * \mathbf{C o}\left(\mathbf{2 , 3}-\mathbf{C}_{2} \mathbf{B}_{4} \mathbf{H}_{5}-5-\mathbf{C H}_{2}\right)\right]_{2}$ (4). In a $50 \mathrm{~mL}$ Schlenk flask under dinitrogen, $167 \mathrm{mg}$ of $\mathrm{Cp} * \mathrm{Co}\left(2,3-\mathrm{C}_{2} \mathrm{~B}_{4} \mathrm{H}_{6}\right)(0.624 \mathrm{mmol})$ was dissolved in $20 \mathrm{~mL}$ of anhydrous. THF. The solution was 
cooled to $-78{ }^{\circ} \mathrm{C}$ and $0.46 \mathrm{~mL}$ of a $1.37 \mathrm{M} t$-BuLi solution $(0.63 \mathrm{mmol})$ was added. After $15 \mathrm{~min}$ the solution was allowed to warm to RT and stir for $1.5 \mathrm{~h}$, after which $85 \mathrm{mg}$ of anhydrous $\mathrm{ZnCl}_{2}$ was transferred into the flask $(0.62 \mathrm{mmol})$. After an additional $1.25 \mathrm{~h}, 36 \mathrm{mg} \mathrm{Pd}\left(\mathrm{PPh}_{3}\right)_{4}(0.031$ mmol) followed by $0.63 \mathrm{~mL}$ of a $1.0 \mathrm{M}$ vinyl bromide solution $(0.63 \mathrm{mmol})$ in THF were added and the reaction was allowed to stir overnight. The solvent was removed in vacuo and the remaining residue was placed atop $3 \mathrm{~cm}$ of silica gel and eluted with $\mathrm{CH}_{2} \mathrm{Cl}_{2}$. The crude material was chromatographed on silica gel TLC plates with a 1:5 $\mathrm{CH}_{2} \mathrm{Cl}_{2}$-hexanes solution affording 81 $\mathrm{mg}$ of recovered starting material and $39 \mathrm{mg}$ of $\mathbf{4}$ as a deep red crystalline solid (22\% yield, $43 \%$ based on starting material consumed). Data for 4: ${ }^{1} \mathrm{H} \mathrm{NMR}\left(\mathrm{CDCl}_{3}\right) \delta 4.99\left(\mathrm{~s}, 4 \mathrm{H},(\mathrm{C} \mathrm{H})_{2} \mathrm{~B}_{4} \mathrm{H}_{3}\right)$, $1.84\left(\mathrm{~s}, 15 \mathrm{H}, \mathrm{C}_{5} M e_{5}\right), 1.38\left(\mathrm{~s}, 4 \mathrm{H}, \mathrm{C}_{2} H_{4}\right) ;{ }^{11} \mathrm{~B} \mathrm{NMR}\left(\mathrm{CDCl}_{3}\right) \delta 23.1\left(\mathrm{~s}, 2 \mathrm{~B}, B-\mathrm{C}_{2} \mathrm{H}_{4}-B\right), 0.4(6 \mathrm{~B}$,

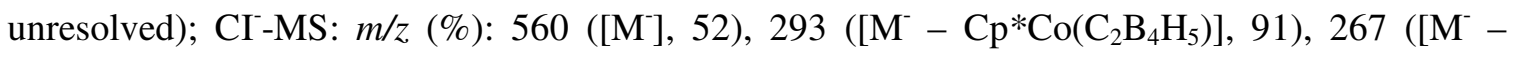
$\left.\left.\mathrm{Cp} * \mathrm{Co}\left(\mathrm{C}_{2} \mathrm{~B}_{4} \mathrm{H}_{5}\right)-5-\mathrm{C}_{2} \mathrm{H}_{4}\right], 100\right)$.

1,3-(Cp*CoEt $\left.{ }_{2} \mathbf{C}_{2} \mathbf{B}_{4} \mathbf{H}_{3}-7-\right)_{2} \mathbf{C}_{6} \mathbf{H}_{4}$ (7a). This compound was obtained as a minor byproduct in the synthesis of the tris(cobaltacarboranyl)benzene complex 8a from 1,3,5tris(dibromoboryl)benzene, described earlier in an analogous procedure using 1,3,5tris(diiodoboryl)benzene. ${ }^{4}$ We assume that $7 \mathbf{a}$ was generated from a small quantity of 1,3bis(dibromoboryl)benzene that was present in the tris(dibromoboryl)benzene reagent. 1,3,5tris(dibromoboryl)benzene was prepared from $6.42 \mathrm{~mL}$ (68 mmol, 4 equiv) of $\mathrm{BBr}_{3}$ which was added to $5 \mathrm{~g}(17 \mathrm{mmol})$ of 1,3,5-tris(trimethylsilyl)benzene, producing a color change from colorless to light brown. The solution was heated for three days at $80{ }^{\circ} \mathrm{C}$, during which the solution turned black. The liquid was removed in vacuo to give dark solid. The crude product was purified by sublimation at 0.4 torr / $135 \mathrm{deg}$. $\mathrm{C}$ to give a colorless, air-sensitive and hygroscopic

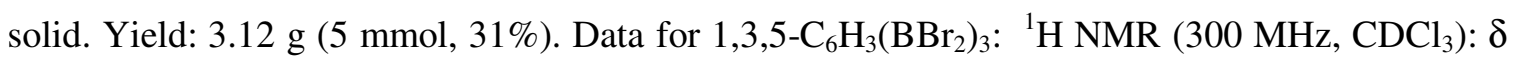
9.30 (s, 3H); ${ }^{11} \mathrm{~B}$ NMR (115.8 MHz, $\left.\mathrm{CDCl} 3\right): \delta 56.6(\mathrm{~s}) ;{ }^{13} \mathrm{C} \mathrm{NMR}\left(75.5 \mathrm{MHz}, \mathrm{CDCl}_{3}\right): \delta 144.3$, 143.3.

Complex 7a was obtained from the reaction of 1,2,3-Cp*Co( $\left.\mathrm{Et}_{2} \mathrm{C}_{2} \mathrm{~B}_{3} \mathrm{H}_{5}\right)(200 \mathrm{mg}, 0.64 \mathrm{mmol})$ in toluene $(30 \mathrm{~mL})$ with $1.6 \mathrm{M} n$-butyllithium $(0.8 \mathrm{~mL}, 1.27 \mathrm{mmol})$ at $0{ }^{\circ} \mathrm{C}$. The solution was warmed to room temperature, after which $188 \mathrm{mg}(0.32 \mathrm{mmol})$ of 1,3,5tris(dibromoboryl)benzene was added under nitrogen, causing the solution to turn brown. The reaction mixture was stirred for $12 \mathrm{~h}$, after which the toluene was removed in vacuo. The residue was taken up in hexane and washed through silica $(3 \mathrm{~cm})$, first with hexane and then with $\mathrm{CH}_{2} \mathrm{Cl}_{2}$, the hexane wash contained only $\left[\mathrm{Cp} * \mathrm{Co}\left(\mathrm{Et}_{2} \mathrm{C}_{2} \mathrm{~B}_{3} \mathrm{H}_{5}\right)\right]$. Column chromatography of the $\mathrm{CH}_{2} \mathrm{Cl}_{2}$ wash on silica with hexane/ $\mathrm{CH}_{2} \mathrm{Cl}_{2}$ (1:4) afforded a few $\mathrm{mg}$ of $\mathbf{7 a}$ as a yellow band. ${ }^{1} \mathrm{H}$ NMR (300 
$\left.\mathrm{MHz}, \mathrm{CDCl}_{3}\right): \delta 6.15-6.60(\mathrm{~m}, 4 \mathrm{H}), 2.29\left(\mathrm{~m}\right.$, ethyl $\left.\mathrm{CH}_{2}\right), 1.78\left(\mathrm{C}_{5} \mathrm{Me}_{5}\right), 1.28$ (t, ethyl $\left.\mathrm{CH}_{3}\right)$. CI+MS: m/z (\%) 723.4 ([M+], 100).

\section{4-17 (see Scheme 1).}

\section{Scheme $1 \bigcirc \mathrm{C}-\mathrm{C}_{2} \mathrm{H}_{5} \bigcirc \mathrm{BH}, \mathrm{B}$}

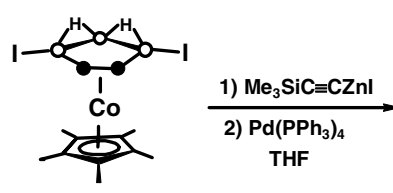

A1

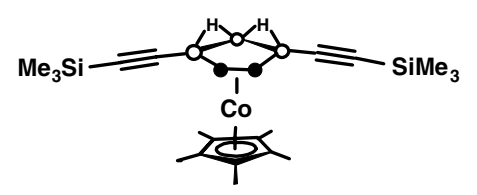

A2

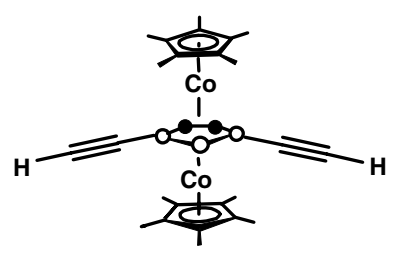

A3
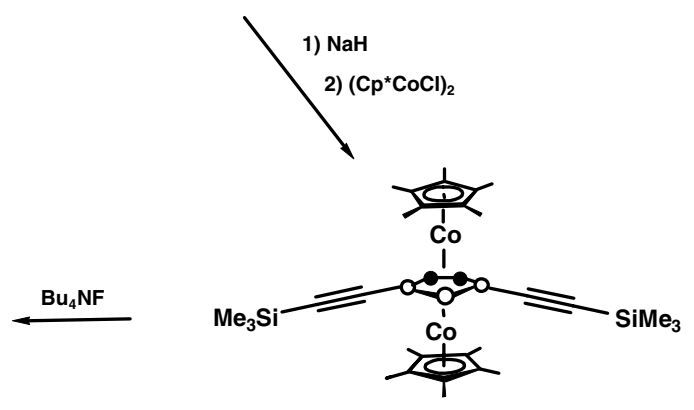

14

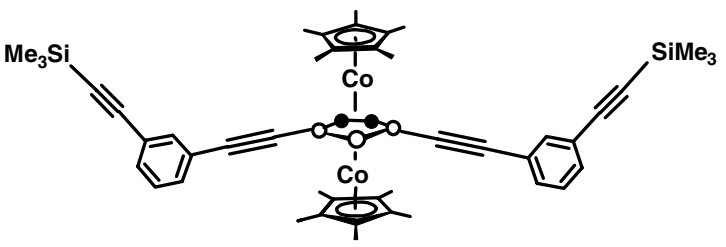

A4
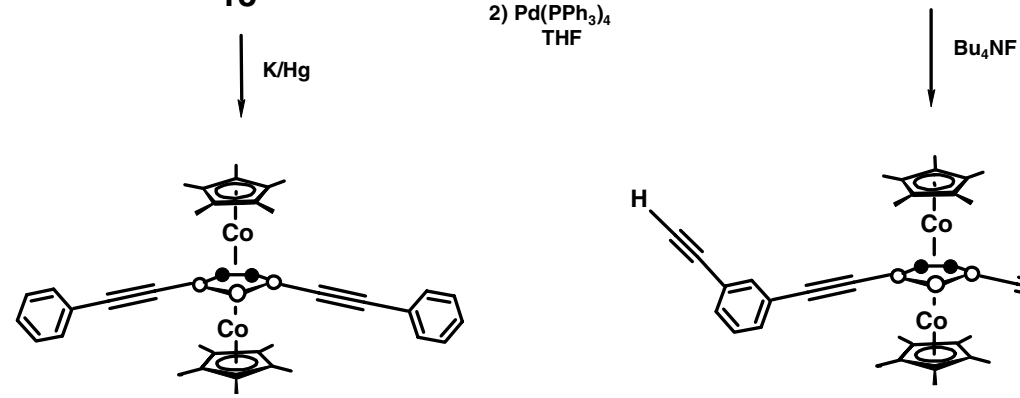

16

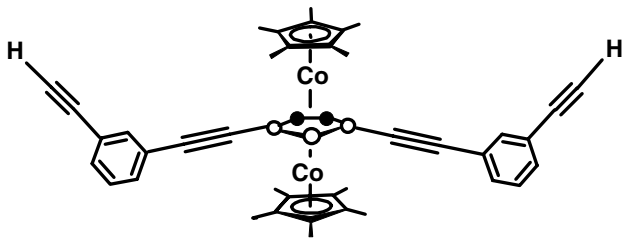

17 
$\mathbf{C p}^{*} \mathrm{Co}\left[2,3-\mathrm{Et}_{2} \mathrm{C}_{2} \mathbf{B}_{3} \mathbf{H}_{3}-\mathbf{4 , 6}-\left(\mathbf{C} \equiv \mathrm{CSSiMe}_{3}\right)_{2}\right] \quad$ (A2). To a solution of $1.37 \mathrm{~g}$ of trimethylsilylacetylene $(14 \mathrm{mmol})$ in $15 \mathrm{ml}$ of THF was added $8.75 \mathrm{ml}$ of a $1.6 \mathrm{M}$ solution of $n$ $\mathrm{BuLi}$ in hexane $(14 \mathrm{mmol})$ at $0^{\circ} \mathrm{C}$. After stirring $0.5 \mathrm{~h}, 4.65 \mathrm{~g}(14 \mathrm{mmol})$ of anhydrous $\mathrm{Znl}_{2}$ in 10 $\mathrm{ml}$ of THF was added at $0^{\circ} \mathrm{C}$ and the mixture was stirred at room temperature for $2 \mathrm{~h} .1 .7 \mathrm{~g}$ (3 mmol) of $\mathbf{A 1}$ and $347 \mathrm{mg}$ of $\mathrm{Pd}\left(\mathrm{PPh}_{3}\right)_{4}(0.3 \mathrm{mmol})$ were added. The mixture was refluxed overnight, following which the solvent was removed in vacuo. The remaining red-brown residue was washed through $5 \mathrm{~cm}$ of silica gel with hexane, followed by $70 \%$ of hexane $/ \mathrm{CH}_{2} \mathrm{Cl}_{2}$ to give one orange band. Removal of solvent gave $1.37 \mathrm{~g}$ of orange-yellow product $(90 \%)$. ${ }^{1} \mathrm{H}$ NMR $\left(500 \mathrm{MHz}, \mathrm{CDCl}_{3}\right.$ ): $\delta-5.34$ (s, 2H, B-H-B), 0.23 (s, 18H, SiMe $), 1.08$ (t, 6H, J = $7.5 \mathrm{~Hz}$, ethyl $\left.\mathrm{CH}_{3}\right), 1.75$ (s, 15H, $\left.\mathrm{C}_{5} \mathrm{Me}_{5}\right), 2.05\left(\mathrm{~m}, 4 \mathrm{H}, J=7.5 \mathrm{~Hz}\right.$, ethyl $\left.\mathrm{CH}_{2}\right) .{ }^{13} \mathrm{C}\left\{{ }^{1} \mathrm{H}\right\} \mathrm{NMR}\left(\mathrm{CDCl}_{3}\right): \delta-0.2$ $\left(\mathrm{SiMe}_{3}\right), 8.5\left(\mathrm{C}_{5} \mathrm{Me}_{5}\right), 16.2$ (ethyl $\left.\mathrm{CH}_{3}\right), 21.3$ (ethyl $\left.\mathrm{CH}_{2}\right), 92.4\left(\mathrm{C}_{5} \mathrm{Me}_{5}\right), 111.8\left(\mathrm{C}_{2} \mathrm{~B}_{3}\right) .{ }^{11} \mathrm{~B} \mathrm{NMR}$ $\left(\mathrm{CDCl}_{3}\right): \delta 0.2$ (s, 2B), 2.9 (BH, 1B, unresolved). IR (KBr pellet, cm-1): v= 2959.8 (s), 2930.6 (m), 2911.1 (m), 2896.9 (w), 2535.0 (s, B-H), 2119.7 (s, C=C), 1836.3 (m, B-H-B), 1486.0 (m), 1467.9 (m), 1492.3 (w), 1384.4 (m), 1247.7 (s), 1126.3 (m), 1081.0 (m), 865.0 (vs), 842.1 (vs), $758.6(\mathrm{~s}), 698.9(\mathrm{~m}), 618.9(\mathrm{~m}) . \mathrm{CI}^{+}-\mathrm{MS}: \mathrm{m} / \mathrm{z}(\%) 507.5\left(\left[\mathrm{M}^{+}\right]+1,100\right)$.

Cp*Co[2,3-Et $\left.{ }_{2} \mathbf{C}_{2} \mathbf{B}_{3} \mathbf{H}-4,6-\left(\mathbf{C} \equiv \mathbf{C S i M e}_{3}\right)_{2}\right] \mathbf{C o C p}^{*}$ (14). $506 \mathrm{mg}$ of A2 (1 mmol) in $8 \mathrm{ml}$ THF was deprotonated with $48 \mathrm{mg}$ of $\mathrm{NaH}(2 \mathrm{mmol})$ at room temperature. This solution was stirred for $2 \mathrm{~h}$, unreactive $\mathrm{NaH}$ was removed by filtration, and $230 \mathrm{mg}$ of $(\mathrm{Cp} * \mathrm{CoCl})_{2}(0.5 \mathrm{mmol})$ in $8 \mathrm{ml}$ THF was added. The mixture was stirred overnight and the solvent was removed in vacuo. The remaining red-brown residue was washed through $5 \mathrm{~cm}$ of silica gel with hexane and then dichloromethane. Removal of the solvent from the dichloromethane wash afforded $662 \mathrm{mg}$ of 14 as an air-stable dark-red solid (95\%). ${ }^{1} \mathrm{H}$ NMR (500MHz, $\left.\mathrm{CDCl}_{3}\right): \delta 0.25\left(\mathrm{~s}, 18 \mathrm{H}, \mathrm{SiMe}_{3}\right)$, $1.63\left(\mathrm{t}, 6 \mathrm{H}, \mathrm{J}=7.5 \mathrm{~Hz}\right.$, ethyl $\left.\mathrm{CH}_{3}\right), 1.64\left(\mathrm{~s}, 30 \mathrm{H}, \mathrm{C}_{5} \mathrm{Me}_{5}\right.$ ), 2.66-2.73 (q, 4H, $J=7.5 \mathrm{~Hz}$, ethyl $\left.\mathrm{CH}_{2}\right) .{ }^{13} \mathrm{C}\left\{{ }^{1} \mathrm{H}\right\}$ NMR $\left(\mathrm{CDCl}_{3}\right): \delta 0.4\left(\mathrm{SiMe}_{3}\right), 9.2\left(\mathrm{C}_{5} \mathrm{Me}_{5}\right), 15.4$ (ethyl $\left.\mathrm{CH}_{3}\right), 25.0$ (ethyl $\left.\mathrm{CH}_{2}\right)$, $85.7\left(\mathrm{C}_{2} \mathrm{~B}_{3}\right), 87.7\left(\mathrm{C}_{5} \mathrm{Me}_{5}\right) .{ }^{11} \mathrm{~B}$ NMR $\left(\mathrm{CDCl}_{3}\right): \delta 3.2(\mathrm{~s}, 2 \mathrm{~B}), 59.5(\mathrm{BH}, 1 \mathrm{~B}$, unresolved). IR ( $\mathrm{KBr}$

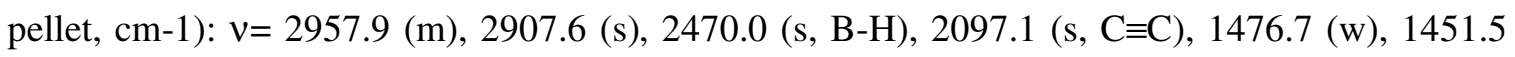
(w), 1476.3 (s), 1245.6 (m), 1121.8 (m), 1026.5 (m), 860.2 (vs), 838.4 (s), 818.4 (s), 755.7 (w), 621.7 (m), 594.5 (w). UV-vis $\left(\mathrm{CH}_{2} \mathrm{Cl}_{2}\right.$, nm (\%): 374 (100), 229 (88), 293 (29), 466 (6) $\varepsilon_{\max }=$ $41,000 \mathrm{~cm}^{-1} \mathrm{M}^{-1}$. CI $I^{+}-\mathrm{MS}: \mathrm{m} / \mathrm{z}(\%) 699.3\left(\left[\mathrm{M}^{+}\right]+1,100\right)$.

Cp*Co[2,3-Et $\left.{ }_{2} \mathbf{C}_{2} \mathbf{B}_{3} \mathbf{H}-4,6-(\mathbf{C} \equiv \mathbf{C H})_{2}\right] \mathrm{CoCp}^{*}$ (A3). $250 \mathrm{mg}$ of $14(0.36 \mathrm{mmol})$ was desilylated with $0.9 \mathrm{~mL}$ of $1.0 \mathrm{M}$ TBAF solution $(0.9 \mathrm{mmol})$ in $6 \mathrm{~mL}$ of dry THF. The reaction mixture was stirred at $0^{\circ} \mathrm{C}$ for $1 \mathrm{~h}$ and then at room temperature for $3 \mathrm{~h}$, following which the THF 
was removed and the reside was flash-chromatographed through $3 \mathrm{~cm}$ of silica gel in $\mathrm{CH}_{2} \mathrm{Cl}_{2}$ to give one red band. Removal of solvent gave $174 \mathrm{mg}$ of product as a dark red solid $(87 \%)$. ${ }^{1} \mathrm{H}$ $\operatorname{NMR}\left(\mathrm{CDCl}_{3}\right): \delta 1.65\left(\mathrm{t}, 6 \mathrm{H}, \mathrm{J}=7.5 \mathrm{~Hz}\right.$, ethyl $\left.\mathrm{CH}_{3}\right), 1.66\left(\mathrm{~s}, 30 \mathrm{H}, \mathrm{C}_{5} \mathrm{Me}_{5}\right), 2.69-2.77(\mathrm{q}, 4 \mathrm{H}, J=$ $7.5 \mathrm{~Hz}$, ethyl $\left.\mathrm{CH}_{2}\right), 3.34(\mathrm{~s}, 2 \mathrm{H}, \mathrm{C} \equiv \mathrm{CH}) .{ }^{13} \mathrm{C}\left\{{ }^{1} \mathrm{H}\right\} \mathrm{NMR}\left(125.75 \mathrm{MHz}, \mathrm{CDCl}_{3}\right): \delta 9.3\left(\mathrm{C}_{5} \mathrm{Me}_{5}\right)$, 15.7 (ethyl $\left.\mathrm{CH}_{3}\right), 24.9$ (ethyl $\left.\mathrm{CH}_{2}\right), 85.8(\mathrm{C} \equiv \mathrm{C}), 87.8\left(\mathrm{C}_{5} \mathrm{Me}_{5}\right), 97.4\left(\mathrm{C}_{2} \mathrm{~B}_{3}\right) .{ }^{11} \mathrm{~B}$ NMR $\left(\mathrm{CDCl}_{3}\right): \delta$ 3.7 (s, 2B), 58.8 (BH, 1B, unresolved ). IR (KBr pellet, $\mathrm{cm}-1): \mathrm{v}=3303.2(\mathrm{~m}, \mathrm{C} \equiv \mathrm{H}), 2970.3(\mathrm{~m})$, 2910.2 (vs), 2476.0 (m, B-H), 2032.5 (w, C $\equiv \mathrm{C}), 1451.5$ (m), 1375.6 (s), 1188.7 (w), 1077.8 (m), 1024.6 (s), 818.4 (m), 623.1 (m), 596.9 (m). UV-vis $\left(\mathrm{CH}_{2} \mathrm{Cl}_{2}, \mathrm{~nm}(\%)\right): 370$ (100), 230 (60), 297 (22), 659 (1) $\varepsilon_{\max }=41,700 \mathrm{~cm}^{-1} \mathrm{M}^{-1}$. CI $\mathrm{I}^{+}-\mathrm{MS}: \mathrm{m} / \mathrm{z}(\%) 555.5\left(\left[\mathrm{M}^{+}\right]+1,100\right)$. Anal. Calcd. for $\mathrm{B}_{3} \mathrm{C}_{30} \mathrm{Co}_{2} \mathrm{H}_{43}$ : C, 65.04; H, 7.82. Found: C, 65.26; H, 7.95.

$\mathbf{C p} * \mathbf{C o}\left\{2,3-\mathbf{E t}_{2} \mathbf{C}_{2} \mathbf{B}_{3} \mathbf{H}-4,6-\left[\mathbf{C} \equiv \mathbf{C}\left(3-\mathrm{BrC}_{6} \mathrm{H}_{4}\right)\right]_{2}\right\} \mathbf{C o C p} *$ (15). $1.5 \mathrm{~mL}$ of triethylamine was added to a stirred mixture of $18 \mathrm{mg} \mathrm{Pd}\left(\mathrm{PPh}_{3}\right)_{4}(0.026 \mathrm{mmol}), 10 \mathrm{mg}$ of $\mathrm{CuI}(0.05 \mathrm{mmol}), 142$ $\mathrm{mg}$ of $\mathbf{A 3}(0.26 \mathrm{mmol})$ and $147 \mathrm{mg}$ of 3-bromo-iodobenzene $(0.52 \mathrm{mmol})$ in $6 \mathrm{~mL}$ of THF under nitrogen. This mixture was stirred for 2 days at room temperature, after which the solvents were removed in vacuo and the residue was flash-chromatographed through $4 \mathrm{~cm}$ of silica gel in $50 \%$ of hexane/ $\mathrm{CH}_{2} \mathrm{Cl}_{2}$ to afford $207 \mathrm{mg}$ of $\mathbf{1 5}$ as a dark red solid (94\%). ${ }^{1} \mathrm{H} \mathrm{NMR}\left(500 \mathrm{MHz}, \mathrm{CDCl}_{3}\right)$ : $\delta 1.69\left(\mathrm{t}, 6 \mathrm{H}, J=7.5 \mathrm{~Hz}\right.$, ethyl $\left.\mathrm{CH}_{3}\right), 1.70\left(\mathrm{~s}, 30 \mathrm{H}, \mathrm{C}_{5} \mathrm{Me}_{5}\right), 2.75-2.79$ (q, 4H, $J=7.5 \mathrm{~Hz}$, ethyl $\mathrm{CH}_{2}$ ), 7.20 (t, 2H, $\left.\mathrm{C}_{6} \mathrm{H}_{4}\right), 7.38,7.40\left(\mathrm{br}, 2 \mathrm{H}, J=10 \mathrm{~Hz}, \mathrm{C}_{6} \mathrm{H}_{4}\right), 7.45,7.47$ (br, $2 \mathrm{H}, J=10 \mathrm{~Hz}$, $\left.\mathrm{C}_{6} \mathrm{H}_{4}\right), 7.64\left(\mathrm{t}, 2 \mathrm{H}, J=2 \mathrm{~Hz}, \mathrm{C}_{6} \mathrm{H}_{4}\right) .{ }^{13} \mathrm{C}\left\{{ }^{1} \mathrm{H}\right\} \mathrm{NMR}\left(125.75 \mathrm{MHz}, \mathrm{CDCl}_{3}\right): \delta 9.4\left(\mathrm{C}_{5} \mathrm{Me}_{5}\right), 15.5$ (ethyl $\left.\mathrm{CH}_{3}\right), 25.1$ (ethyl $\left.\mathrm{CH}_{2}\right), 88.1\left(\mathrm{C}_{5} \mathrm{Me}_{5}\right), 109.2\left(\mathrm{C}_{2} \mathrm{~B}_{3}\right), 122.1\left(\mathrm{C}_{6} \mathrm{H}_{4}\right), 129.0\left(\mathrm{C}_{6} \mathrm{H}_{4}\right), 129.2$ $\left(\mathrm{C}_{6} \mathrm{H}_{4}\right), 129.3\left(\mathrm{C}_{6} \mathrm{H}_{4}\right), 129.6\left(\mathrm{C}_{6} \mathrm{H}_{4}\right), 133.1\left(\mathrm{C}_{6} \mathrm{H}_{4}\right) .{ }^{11} \mathrm{~B}$ NMR $\left(\mathrm{CDCl}_{3}\right)$ : $\delta 3.3(\mathrm{~s}, 2 \mathrm{~B}), 59.3(\mathrm{BH}$, 1B, unresolved ). IR (KBr pellet, cm-1): v = 3057.5 (w), 2976.7 (m), 2902.9 (s), 2568.9 (m, B-H),

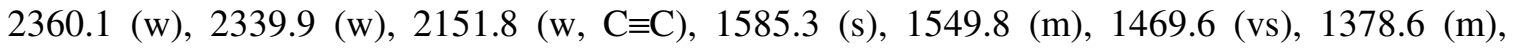
1096.4 (m), 1066.6 (m), 1029.7 (m), 812.6 (m), 775.1 (s), 676.3 (m). UV-vis $\left(\mathrm{CH}_{2} \mathrm{Cl}_{2}, \mathrm{~nm}(\%)\right)$ : 253 (100), 376 (97), 309 (71) $\varepsilon_{\max }=35,893 \mathrm{~cm}^{-1} \mathrm{M}^{-1}$. CI ${ }^{+}-\mathrm{MS}: \mathrm{m} / \mathrm{z}(\%)=864.4\left(\left[\mathrm{M}^{+}\right]+2,100\right)$.

Cp*Co[2,3-Et $\left.\mathbf{C}_{2} \mathbf{B}_{3} \mathbf{H}-4,6-\left(\mathbf{C} \equiv \mathbf{C C}_{6} \mathbf{H}_{4}\right)_{2}\right] \mathbf{C o C p} *$ (16). In a $50 \mathrm{~mL}$ Schlenk flask under nitrogen, $11 \mathrm{mg}$ potassium metal $(0.27 \mathrm{mmol})$ was dissolved in $1.2 \mathrm{~mL}$ clean mercury. $6 \mathrm{~mL}$ of THF followed by $117 \mathrm{mg}(0.135 \mathrm{mmol})$ of $\mathbf{1 5}$ was added at $-20{ }^{\circ} \mathrm{C}$. The reaction mixture was stirred at room temperature overnight and separated from the mercury, and the solvent was removed in vacuo. The remaining residue was placed atop $3 \mathrm{~cm}$ of silica gel and eluted with $\mathrm{CH}_{2} \mathrm{Cl}_{2}$. The crude material was chromatographed on silica gel TLC plates with a 3:2 $\mathrm{CH}_{2} \mathrm{Cl}_{2}-$ hexanes solution, affording $32 \mathrm{mg}$ of $\mathbf{1 6}$ and $8 \mathrm{mg}$ of recovered $\mathbf{1 5}$ (33\% yield, 36\% based on $\mathbf{9}$ 
consumed). ${ }^{1} \mathrm{H}$ NMR $\left(500 \mathrm{MHz}, \mathrm{CDCl}_{3}\right): \delta 1.71\left(\mathrm{t}, 6 \mathrm{H}, J=7.5 \mathrm{~Hz}\right.$, ethyl $\left.\mathrm{CH}_{3}\right), 1.72(\mathrm{~s}, 30 \mathrm{H}$, $\mathrm{C}_{5} \mathrm{Me}_{5}$ ), 2.76-2.81 (q, 4H, $J=7.5 \mathrm{~Hz}$, ethyl $\left.\mathrm{CH}_{2}\right), 7.26\left(\mathrm{t}, 2 \mathrm{H}, J=7 \mathrm{~Hz}, \mathrm{C}_{6} \mathrm{H}_{4}\right), 7.35(\mathrm{t}, 4 \mathrm{H}, J=9$ $\left.\mathrm{Hz}, \mathrm{C}_{6} \mathrm{H}_{4}\right), 7.54,7.56$ (br, 4H, $\left.J=10 \mathrm{~Hz}, \mathrm{C}_{6} \mathrm{H}_{4}\right) .{ }^{13} \mathrm{C}\left\{{ }^{1} \mathrm{H}\right\} \mathrm{NMR}\left(125.75 \mathrm{MHz}, \mathrm{CDCl}_{3}\right): \delta 9.4$ $\left(\mathrm{C}_{5} \mathrm{Me}_{5}\right.$ ), 15.6 (ethyl $\left.\mathrm{CH}_{3}\right), 25.1$ (ethyl $\left.\mathrm{CH}_{2}\right), 88.0\left(\mathrm{C}_{5} \mathrm{Me}_{5}\right), 110.7\left(\mathrm{C}_{2} \mathrm{~B}_{3}\right), 126.2\left(\mathrm{C}_{6} \mathrm{H}_{4}\right), 127.2$ $\left(\mathrm{C}_{6} \mathrm{H}_{4}\right), 128.2\left(\mathrm{C}_{6} \mathrm{H}_{4}\right), 130.5\left(\mathrm{C}_{6} \mathrm{H}_{4}\right) .{ }^{11} \mathrm{~B}$ NMR $\left(\mathrm{CDCl}_{3}\right)$ : $\delta 4.0(\mathrm{~s}, 2 \mathrm{~B}), 59.5$ (BH, 1B, unresolved). IR (KBr pellet, cm-1): v = 3058.0 (w), 2974.6 (m), 2901.9 (vs), 2468.4 (m, B-H), 2366.9 (w),

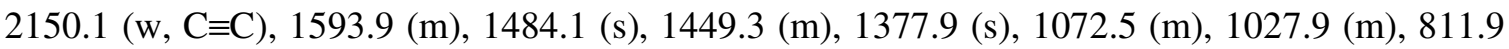
(m), 754.0 (s), 689.9 (m). UV-vis $\left(\mathrm{CH}_{2} \mathrm{Cl}_{2}, \mathrm{~nm}(\%)\right): 249$ (100), 376 (98), 308 (66) $\varepsilon_{\max }=36,813$ $\mathrm{cm}^{-1} \mathrm{M}^{-1}$. CI ${ }^{+}-\mathrm{MS}: \mathrm{m} / \mathrm{z}(\%) 707.4\left(\left[\mathrm{M}^{+}\right]+1,100\right)$. Anal. Calcd. for $\mathrm{B}_{3} \mathrm{C}_{42} \mathrm{H}_{51} \mathrm{Co}_{2}: \mathrm{C}, 71.44 ; \mathrm{H}, 7.28$. Found: C, 71.21; H, 7.27.

$\mathrm{Cp} * \mathrm{Co}\left\{2,3-\mathrm{Et}_{2} \mathrm{C}_{2} \mathrm{~B}_{3} \mathrm{H}-4,6-\left[\mathrm{C} \equiv \mathrm{C}\left(3-\mathrm{TMSC} \equiv \mathrm{CC}_{6} \mathrm{H}_{4}\right)\right]_{2}\right\} \mathrm{CoCp} *$ (A4). To a solution of $118 \mathrm{mg}$ of trimethylsilylacetylene $(1.2 \mathrm{mmol})$ in $3 \mathrm{ml}$ of THF was added $0.75 \mathrm{ml}$ of $1.6 \mathrm{M}$ solution of $n$-BuLi in hexane $(1.2 \mathrm{mmol})$ at $0^{\circ} \mathrm{C}$. After $0.5 \mathrm{~h}$ of stirring, $164 \mathrm{mg}(1.2 \mathrm{mmol})$ of anhydrous $\mathrm{ZnCl}_{2}$ in $2 \mathrm{ml}$ of $\mathrm{THF}$ was added at $0^{\circ} \mathrm{C}$ and the mixture was stirred at room temperature for $2 \mathrm{~h}$. followed by $173 \mathrm{mg}$ of $15(0.2 \mathrm{mmol})$ and $23 \mathrm{mg}$ of $\mathrm{Pd}\left(\mathrm{PPh}_{3}\right)_{4}(0.02 \mathrm{mmol})$ were added. The mixture was refluxed overnight, after which the solvents were removed in vacuo and the reside was flash-chromatographed through $5 \mathrm{~cm}$ of silica gel in hexane and then $30 \%$ of hexane $/ \mathrm{CH}_{2} \mathrm{Cl}_{2}$ to give one red band. Removal of solvent gave $175 \mathrm{mg}$ of dark red product (97 \%). ${ }^{1} \mathrm{H}$ NMR (500 MHz, $\mathrm{CDCl}_{3}$ ): $\delta 0.30\left(\mathrm{~s}, 18 \mathrm{H}, \mathrm{SiMe}_{3}\right), 1.72\left(\mathrm{t}, 6 \mathrm{H}, J=7.5 \mathrm{~Hz}\right.$, ethyl $\left.\mathrm{CH}_{3}\right), 1.72$ (s, 30H, $\mathrm{C}_{5} \mathrm{Me}_{5}$ ), 2.63-2.95 (q, 4H, J = 7.5 Hz, ethyl $\mathrm{CH}_{2}$ ), 7.29 (t, $2 \mathrm{H}, \mathrm{C}_{6} \mathrm{H}_{4}, J=7.5 \mathrm{~Hz}$,), 7.37, 7.39 (br, 2H, $\left.J=10 \mathrm{~Hz}, \mathrm{C}_{6} \mathrm{H}_{4}\right), 7.50,7.52\left(\mathrm{br}, 2 \mathrm{H}, J=10 \mathrm{~Hz}, \mathrm{C}_{6} \mathrm{H}_{4}\right), 7.62(\mathrm{t}, 2 \mathrm{H}, J=1.5 \mathrm{~Hz}$, $\left.\mathrm{C}_{6} \mathrm{H}_{4}\right) \cdot{ }^{13} \mathrm{C}\left\{{ }^{1} \mathrm{H}\right\}$ NMR $\left(125.75 \mathrm{MHz}, \mathrm{CDCl}_{3}\right): \delta 0.0\left(\mathrm{SiMe}_{3}\right), 9.5\left(\mathrm{C}_{5} \mathrm{Me}_{5}\right), 15.6$ (ethyl $\left.\mathrm{CH}_{3}\right), 25.1$ (ethyl $\left.\mathrm{CH}_{2}\right), 88.1\left(\mathrm{C}_{5} \mathrm{Me}_{5}\right), 94.2(\mathrm{C} \equiv \mathrm{C}), 105.0(\mathrm{C} \equiv \mathrm{C}), 109.8\left(\mathrm{C}_{2} \mathrm{~B}_{3}\right), 123.1\left(\mathrm{C}_{6} \mathrm{H}_{4}\right), 127.2\left(\mathrm{C}_{6} \mathrm{H}_{4}\right)$, $128.2\left(\mathrm{C}_{6} \mathrm{H}_{4}\right), 129.9\left(\mathrm{C}_{6} \mathrm{H}_{4}\right), 130.9\left(\mathrm{C}_{6} \mathrm{H}_{4}\right), 133.7\left(\mathrm{C}_{6} \mathrm{H}_{4}\right) .{ }^{11} \mathrm{~B}$ NMR $\left(\mathrm{CDCl}_{3}\right): \delta 3.3(\mathrm{~s}, 2 \mathrm{~B}), 60.0$ (BH, 1B, unresolved ). IR (KBr pellet, cm-1): v = 2960.5 (m), 2903.5 (s), 2471.3 (m, B-H), 2360.9 (m), 2340.3 (w), 2156.6 (m, C $\equiv C), 1588.9$ (m), 1563.9 (w), 1473.9 (s), 1380.2 (m), 1249.3 (m), 1180.7 (w), 1028.1 (w), 845.4 (vs), 791.5 (m), 686.3 (w). CI'-MS: m/z (\%) 899.5 ([M+1 $]+1$, 100).

$\mathbf{C p} * \mathbf{C o}\left\{2,3-\mathrm{Et}_{2} \mathbf{C}_{2} \mathbf{B}_{3} \mathbf{H}-4,6-\left[\mathbf{C} \equiv \mathbf{C}\left(3-\mathbf{H C} \equiv \mathbf{C C}_{6} \mathbf{H}_{4}\right)\right]_{2}\right\} \mathbf{C o C p}^{*}(\mathbf{1 7}) .156 \mathrm{mg}$ of $\mathbf{A 4}(0.17$ mmol) was desilylated with $0.38 \mathrm{ml}$ of $1.0 \mathrm{M}$ TBAF solution $(0.38 \mathrm{mmol})$ in $8 \mathrm{ml}$ of dry THF. The reaction mixture was stirred at $0^{\circ} \mathrm{C}$ for $1 \mathrm{~h}$, following which the THF was removed and the reside was flash-chromatographed through $3 \mathrm{~cm}$ of silica gel in $\mathrm{CH}_{2} \mathrm{Cl}_{2}$ to give one orange red 
band. Removal of solvent gave $115 \mathrm{mg}$ of $\mathbf{1 7}$ as a dark red solid $(90 \%)$. ${ }^{1} \mathrm{H}$ NMR $(500 \mathrm{MHz}$, $\mathrm{CDCl}_{3}$ ): $\delta 1.71\left(\mathrm{t}, 6 \mathrm{H}, J=7.5 \mathrm{~Hz}\right.$, ethyl $\left.\mathrm{CH}_{3}\right), 1.71$ (s, 30H, $\mathrm{C}_{5} \mathrm{Me}_{5}$ ), 2.76-2.80 (q, 4H, J=7.2 Hz, ethyl $\mathrm{CH}_{2}$ ), 7.30 (t, $2 \mathrm{H}, J=7.5 \mathrm{~Hz}, \mathrm{C}_{6} \mathrm{H}_{4}$ ), 7.38, 7.40 (br, $2 \mathrm{H}, J=10 \mathrm{~Hz}, \mathrm{C}_{6} \mathrm{H}_{4}$ ), 7.52, 7.54 (br, $\left.2 \mathrm{H}, J=10 \mathrm{~Hz}, \mathrm{C}_{6} \mathrm{H}_{4}\right), 7.65\left(\mathrm{t}, 2 \mathrm{H}, J=1.5 \mathrm{~Hz}, \mathrm{C}_{6} \mathrm{H}_{4}\right),{ }^{13} \mathrm{C}\left\{{ }^{1} \mathrm{H}\right\} \mathrm{NMR}\left(125.75 \mathrm{MHz}, \mathrm{CDCl}_{3}\right): \delta$ $9.4\left(\mathrm{C}_{5} \mathrm{Me}_{5}\right), 15.6$ (ethyl $\left.\mathrm{CH}_{3}\right), 25.1$ (ethyl $\left.\mathrm{CH}_{2}\right), 77.1(\mathrm{C} \equiv \mathrm{C}), 83.5(\mathrm{C} \equiv \mathrm{C}), 88.1\left(\mathrm{C}_{5} \mathrm{Me}_{5}\right), 109.6$ $\left(\mathrm{C}_{2} \mathrm{~B}_{3}\right), 122.1\left(\mathrm{C}_{6} \mathrm{H}_{4}\right), 127.3\left(\mathrm{C}_{6} \mathrm{H}_{4}\right), 128.3\left(\mathrm{C}_{6} \mathrm{H}_{4}\right), 129.9\left(\mathrm{C}_{6} \mathrm{H}_{4}\right), 131.1\left(\mathrm{C}_{6} \mathrm{H}_{4}\right), 133.9\left(\mathrm{C}_{6} \mathrm{H}_{4}\right) .{ }^{11} \mathrm{~B}$ NMR $\left(\mathrm{CDCl}_{3}\right): \delta 3.5(\mathrm{~s}, 2 \mathrm{~B}), 62.6(\mathrm{BH}, 1 \mathrm{~B}$, unresolved ). IR (KBr pellet, $\mathrm{cm}-1): \mathrm{v}=3305.0(\mathrm{w}$, $\mathrm{C} \equiv \mathrm{CH}), 3256.5$ (s, C $\equiv \mathrm{CH}), 3054.6$ (w), 2972.7 (m), 2904.5 (s), 2457.9 (m, B-H), 2360.6 (w), 2143.0 (m, C $\equiv \mathrm{C}), 1588.8$ (s), 1565.6 (m), 1473.6 .6 (vs), 1376.1 (s), 1026.7 (m), 891.6 (m), 791.6 (vs), 685.6 (m). UV-vis $\left(\mathrm{CH}_{2} \mathrm{Cl}_{2}\right.$, nm (\%)): 237 (100), 376 (56) $\varepsilon_{\max }=68,600 \mathrm{~cm}^{-1} \mathrm{M}^{-1}$. CI ${ }^{+}-\mathrm{MS}$ : $\mathrm{m} / \mathrm{z}(\%) 755.5\left(\left[\mathrm{M}^{+}\right]+2,100\right)$.

\section{References:}

1. Davis, J. H., Jr.; Sinn, E.; Grimes, R. N., J. Am. Chem. Soc. 1989, 111, 4776.

2. Stockman, K. E.; Garrett, D. L.; Grimes, R. N., Organometallics, 1995, 14, 4661

3. Koelle, U.; Fuss, B.; Belting, M.; Raabe, E., Organometallics, 1986, 5, 980.

4. Bluhm, M.; Pritzkow, H.; Siebert, W.; Grimes, R. N., Angew. Chem. Int. Ed. 2000, 39, 4562. 